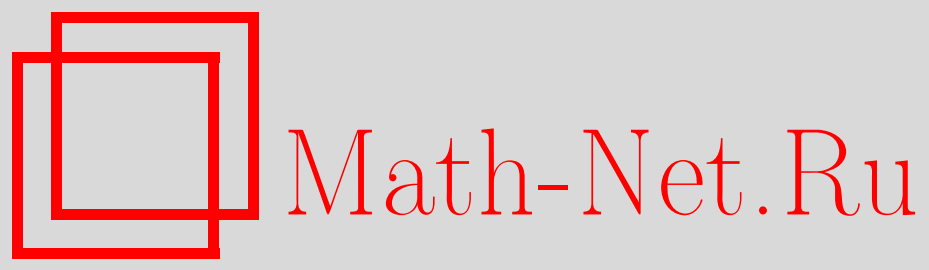

А. М. Вершик, С. В. Керов, Об одной бесконечномерной группе над конечным полем, Функи. анализ и его прил., 1998, том 32, выпуск 3, 3-10

DOI: https://doi.org/10.4213/faa418

Использование Общероссийского математического портала MathNet.Ru подразумевает, что вы прочитали и согласны с пользовательским соглашением

http://www . mathnet.ru/rus/agreement

Параметры загрузки:

IP : 54.237 .59 .107

26 апреля 2023 г., 02:33:30

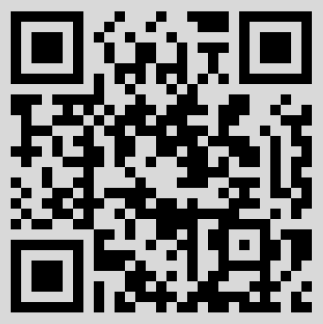


Функииональньй анализ и еәо приложения

1998, т. 32, вып. 3, с. 3-10

УдК $512.743,517.986,519.217$

\section{Об одной бесконечномерной группе над конечным полем ${ }^{\star}$}

(C) 1998. А. М. ВеРшик, С. В. КеРов

Введение. Асимптотическая теория представлений изучает структуру представлений классических групп высоких степеней и их бесконечномерных аналогов. Одним из основных примеров служит серия линейных групп $G L_{n}\left(\mathbb{F}_{q}\right)$ над конечным полем $k=\mathbb{F}_{q}$ и их индуктивный предел $G L_{\infty}\left(\mathbb{F}_{q}\right)$ группа финитных обратимых матриц над полем $k$.

В этой заметке вводится новая локально компактная группа $G L B$ и изучается ее структура, характеры и непрерывные унитарные представления. Эта группа содержит группу $G L_{\infty}(k)$ в качестве счетной плотной подгруппы. Ценность группы $G L B$ определяется ее тесными связями с теорией параболического индуцирования - ядром теории представлений групп $G L_{n}(k)$.

Описание неприводимых представлений конечных групп $G L_{n}(k)$ впервые получено в работе Грина [6]. Основным инструментом его теории, развитым в дальнейшем в работах $[5,10]$, служит операция параболического индуцирования представлений. Именно эта операция определяет те вложения групповых алгебр $\mathbb{C}\left(G L_{n}(k)\right)$, которые приводят к интересующей нас групповой алгебре $A$ группы $G L B$. Алгебра $A$ представима как индуктивный предел алгебр $\mathbb{C}\left(G L_{n}(k)\right)$, хотя вложения этих групповых алгебр уже не порождаются вложениями групп, а задаются операторами усреднения по классам смежности относительно некоторой подгруппы.

Группа $G L B$ была определена авторами настоящей работы совместно с А. В. Зелевинским (см. добавление редактора перевода в книге [3]) в 1981 г. в процессе обсуждения связей его работы [10] с теорией представлений и характеров локально конечных групп (подобных $G L_{\infty}(k)$ и бесконечной симметрической группе $\left.\mathfrak{S}_{\infty}\right)$.

Применяемый метод исследования опирается на общую теорию индуктивных пределов конечномерных полупростых алгебр. Заметим, что при $q=1$ мы приходим к теории представлений бесконечной симметрической группы, рассмотренной в ряде работ авторов (см. например ссылки в [1]). То замечательное обстоятельство, что бесконечномерная недискретная группа $G L B$ локально компактна, позволяет в полной мере использовать классические методы теории представлений, в первую очередь метод индуцированных представлений. В следующих работах мы собираемся рассмотреть более подробно унипотентные характеры группы $G L B$ и дать реализации факторпредставлений групп $G L B$ и $G L_{\infty}(k)$.

\footnotetext{
* Работа выполнена при поддержке РФФИ, грант 96-01-00676.
} 
1. Группа $G L B$. Определяемые ниже объекты зависят от числа $q$ элементов поля, но для удобства обозначений мы не всегда делаем эту зависимость явной.

Рассмотрим $k$-линейное пространство $V=k_{\infty}$ всех финитных векторов с координатами из конечного поля $k=\mathbb{F}_{q}$. Зафиксируем базис ортов $\left\{e_{i}, i=1,2, \ldots\right\}$ в пространстве $V$ и обозначим через $V_{n}$ подпространство, порожденное первыми $n$ ортами.

ОПРЕДЕЛЕНИЕ. Группа $G L B$ состоит из $k$-линейных преобразований пространства $V$, сохраняющих почти все подпространства $V_{n}$.

Переформулируем определение в терминах матриц операторов $g \in G L B$ по отношению к выделенному базису. Бесконечную матрицу $g=\left(g_{i j}\right), i, j=$ $1,2, \ldots$, назовем почти треугольной, если число ненулевых поддиагональных элементов $a_{i j} \neq 0, i>j$, конечно. Группа $G L B$ состоит в точности из операторов с почти треугольными матрицами.

2. Борелевская подгруппа. Пусть $G(m, \infty) \subset G L B$ - подгруппа операторов в пространстве $V$, сохраняющих подпространства $V_{n}$ при всех $m \leqslant n$. Если $m \leqslant n$, то через $G(m, n) \subset G L_{n}(k)$ обозначим параболическую подгруппу операторов в $V_{n}$, сохраняюших подпространства $V_{s}$ при $m \leqslant s \leqslant n$. Группа $G(m, \infty) \cong \lim _{\longleftarrow} G(m, n)$ является проконечной группой и в топологии проективного предела вполне несвязна и компактна. Базисом окрестностей единицы в $G(m, \infty)$ служит семейство подгрупп $N(n, \infty)$, $n \geqslant m$, где через $N(n, \infty)$ мы обозначаем группу операторов в $V$, coхраняющих подпространства $V_{n}, V_{n+1}, \ldots$ и тривиальных (единичных) на $V_{n}$. На матричном языке $G(m, \infty)$ описывается как группа бесконечных матриц $g=\left(g_{i j}\right)$ с нулевыми поддиагональными элементами ниже $m$-й строки, т.е. $g_{i j}=0$ при $i>\max (j, m)$.

При $m<n$ группа $G(m, \infty)$ является подгруппой конечного индекса в $G(n, \infty)$. Группа $G L B=\underset{\lim }{\longrightarrow} G(m, \infty)$ есть объединение (индуктивный предел) компактных групп $G \overrightarrow{(m}, \infty)$. Топология на группе $G L B$, как и на подгруппах $G(m, \infty)$, определяется базисом окрестностей единицы, состоящим из подгрупп $N(n, \infty)$. В этой топологии $G L B$ вполне несвязна, локально компактна и является бесконечномерной группой Ли над полем $k$. Очевидно, что $G L_{\infty}(k)$ есть плотная подгруппа в $G L B$. Замыкание любой конечно порожденной подгруппы в $G L B$ компактно, а факторгруппа $P G L B \cong G L B / k^{*}$ по центру группы $G L B$ топологически проста.

Группа $B(n)=G(1, n)$ верхнетреугольных матриц является борелевской подгруппой в $G L_{n}(k)$. Поэтому проконечную группу $B=G(1, \infty)$ естественно считать борелевской подгруппой в $G L B$. Она состоит из операторов в пространстве $V$, сохраняющих все подпространства $V_{n}, n=1,2, \ldots$. Матрицы операторов из $B$ - это все обратимые верхнетреугольные матрицы. Как топологическое пространство группа $B$ есть бесконечное произведение подмножеств в конечном поле $k$, а потому компактна и вполне несвязна. Мера Хаара на $B$ есть продакт-мера с равномерными мерами на сомножителях. Непосредственно из определений вытекает

Теорема 1. Группа GLB с описанной выле топологией является локально компактной унимодулярной аменабельной группой.

Зафиксируем меру Хаара $\mu$ на $G L B$ с нормировкой $\mu(B)=1$. 
3. Групповая алгебра Брюа-Шварца. Определяемая в этом пункте важная подалгебра $A$ групповой алгебры $L^{1}(G L B, \mu)$ является аналогом пространства функций Брюа-Шварца в теории $p$-адических линейных групп, и мы сохраняем за ней то же название. В алгебре всех комплексных непрерывных функций с компактным носителем на группе $G L B$ и обычными операциями свертки и инволюции определим *-подалгебру $A$, состоящую из функций, принимающих конечное число значений. Заметим, что алгебра $A$ плотна в $L^{1}(G L B, \mu)$. Мы покажем, что $A$ локально полупроста, т. е. представима как объединение возрастающей цепочки конечномерных полупростых алгебр над полем $\mathbb{C}$.

ТЕорема 2. Алгебра $А$ есть локально полупростая *-алгебра без единuцbl.

Пусть $A_{n} \subset A$ - подалгебра функций с носителем в компактной подгруппе $G(n, \infty)$, зависящих только от ограничения оператора $g \in G(n, \infty)$ на подпространство $V_{n}$. Алгебра $A_{n}$ конечномерна, полупроста и изоморфна групповой алгебре $\mathbb{C}\left(G_{n}\right)$ конечной группы $G_{n}=G L_{n}(k)$. Для заданного $g \in G_{n}$ обозначим через $\tilde{a}_{g} \in A_{n}$ характеристическую функцию множества операторов $h \in G(n, \infty)$ в пространстве $V$, сохраняющих подпространства $V_{n}, V_{n+1}, \ldots$ и совпадающих с $g$ при ограничении на $V_{n}$. Положим $a_{g}=|B / N(n, \infty)| \tilde{a}_{g}=(q-1)^{n} q^{\left(\begin{array}{c}n \\ 2\end{array}\right)} \tilde{a}_{g}$. Тогда функции $a_{g}, g \in G_{n}$, образуют линейный базис в алгебре $A_{n}$, причем $a_{g h}=a_{g} a_{h}$ и $a_{g}^{*}=a_{g^{-1}}$. Элемент $a_{e}$ (где $e$ - единичный оператор в $V_{n}$ ) служит единицей в алгебре $A_{n}$.

Алгебра $A=\bigcup A_{n}$ есть объединение вложенных подалгебр $A_{n}$. Поскольку элемент $a_{e}$ не является единицей в большей алгебре $A_{n+1}$, алгебра $A$ не содержит единицы.

Хотя алгебра $A$ представлена выше как объединение групповых алгебр, их вложения не порождаются вложениями самих групп. Более точно, вложение $i: A_{n} \rightarrow A_{n+1}$ задается операцией усреднения по подгруппе,

$$
i\left(a_{g}\right)=\frac{1}{\left|N_{n}\right|} \sum_{h \in N_{n}} a_{g h}, \quad g \in G_{n},
$$

где $N_{n}=G_{n+1} \cap N(n, \infty)$ - подгруппа операторов в $V_{n+1}$, единичных на подпространстве $V_{n}$. В базисе $\left\{\tilde{a}_{g}\right\}$ вложения задаются формулой $i\left(\tilde{a}_{g}\right)=$ $\sum_{h \in N_{n}} \tilde{a}_{g h}$.

Строение возрастающей цепочки $A_{1} \subset A_{2} \subset \ldots \subset A_{n} \subset \ldots$ конечномерных полупростых алгебр, а следовательно, и предельной алгебры $A=\bigcup A_{n}$, полностью определяется ее графом ветвления (диаграммой Браттели), описывающим ветвление неприводимых представлений алгебр $A_{n+1}$ при их ограничении на младшую подалгебру $A_{n}$, и размерностлми неприводимьх представлений (см., например, [1]). Вершинами графа ветвления служат классы эквивалентности неприводимых представлений всех алгебр $A_{n}$ или, что то же самое, всех групп $G L_{n}(k)$. Поскольку вложения алгебр не сохраняют единицу, ограничение ненулевого представления алгебры $A_{n+1}$ на подалгебру $A_{n}$ может содержать нулевое представление этой алгебры.

4. Схема ветвления семейства алгебр $\boldsymbol{A}_{\boldsymbol{n}}$. Начнем с более общей конструкции, подготавливающей описание диаграммы Браттели индуктивного семейства алгебр $A_{n}$. 
Пусть $C=\bigcup_{d=1}^{\infty} C_{d}$ - некоторое градуированное семейство конечных множеств и $e \in C_{1}$ - выделенная точка. Число $d=d(c)$ назовем cmeneнью элемента $c \in C_{d}$. Обозначим через $\mathbb{Y}=\bigcup_{n=0}^{\infty} \mathbb{Y}_{n}$ множество диаграмм Юнга, и пусть $|\lambda|=n$ - число клеток в диаграмме $\lambda \in \mathbb{Y}_{n}$. Семейством диаграмм Юнга над $C$ назовем любую функцию $\varphi: C \rightarrow \mathbb{Y}$ с конечным числом непустых значений $\varphi(c) \neq \varnothing$. Введем градуировку семейств, принимая за степень семейства число $|\varphi| \equiv \sum_{c \in C}|\varphi(c)| d(c)$. Пусть $\Phi_{n}$ - множество семейств степени $|\varphi|=n$, а $\Phi=\bigcup_{n=0}^{\infty} \Phi_{n}$ - совокупность всех семейств. Мы берем $\Phi$ за множество вершин определяемого ниже градуированного графа. Его дуги определяются так: два заданных семейства $\varphi \in \Phi_{n}, \psi \in \Phi_{n+1}$ соединены дугой (условимся писать $\varphi \nearrow \psi)$, если $\varphi(c)=\psi(c)$ при всех $c \neq e$, а диаграмма Юнга $\psi(e)$ получается из $\varphi(e)$ добавлением одной клетки. Тем самым определен некоторый граф ветвления (диаграмма Браттели) Ф. Элементы множества $C$ естественно отождествляются с семействами, в которых единственная непустая диаграмма одноклеточна. Такие семейства мы называем каспидальньлми.

Заметим, что диаграмма $\Phi$ несвязна и распадается в объединение компонент, изоморфных графу Юнга. Компоненты связности параметризуются своими начальньли семействами $\varphi$, для которых диаграмма $\varphi(e)=\varnothing$ пуста. Множество начальных семейств условимся обозначать через $\widetilde{\Phi}$.

Искомый граф ветвления индуктивного семейства алгебр $\left\{A_{n}\right\}$ получается при специальном выборе множества $C=C(q)$, зависящем от $q$. Для наших целей достаточно следующего комбинаторного способа описания множества $C$. Зафиксируем натуральное число $q$. При $d \geqslant 2$ пусть $C_{d}(q)-$ множество слов Линдона длины $d$ в алфавите $\{0,1, \ldots, q-1\}$ из $q$ букв. Напомним, что слово называется словом Линдона, если оно лексикографически строго меньше всех своих циклических сдвигов. Известно, что $\left|C_{d}(q)\right|=$ $\sum_{m \mid d} \mu(d / m) q^{m} / d$, где $\mu$ - классическая функция Мёбиуса. При $d=1$ положим $C_{1}(q)=\{1, \ldots, q-1\}$ (т.е. слово 0 исключено) и возьмем $e=1$ в качестве выделенного элемента. Обозначим через $\Phi=\Phi(q)$ граф ветвления семейств диаграмм Юнга, построенный по градуированному множеству $C=\bigcup C_{d}(q)$

ТЕОрема 3. Пусть $q$ - степень простого числа. Тогда граф ветвления $\Phi$ является диаграммой Браттели семейства подалгебр $A_{1} \subset A_{2} \subset \ldots \subset$ $A_{n} \subset \ldots$, , аппроксимируюших групповую алгебру $A$ группь $G L B$. Это означает, что характерьл $\chi_{\varphi}$ неприводимьх представлений $\pi_{\varphi}$ алгебрь $A_{n}$ nараметризуются семействами $\varphi \in \Phi_{n}$ степени $n$, а ограничение характера неприводимого представления $\chi_{\psi}$ алгебры $A_{n+1}$ на подалгебру $A_{n}$ разлагается в свободную от кратностей сумму

$$
\frac{1}{\left|N_{n}\right|} \sum_{h \in N_{n}} \chi_{\psi}\left(a_{g h}\right)=\sum_{\varphi: \varphi \nearrow \psi} \chi_{\varphi}\left(a_{g}\right), \quad g \in G_{n} .
$$

Напомним параметризацию неприводимых представлений линейных групп $G_{n}=G L_{n}(k)$ над конечным полем (см. $\left.[5,6,10]\right)$. Ключевую роль здесь играет следующая операция параболического индуиирования. Если $\pi_{1}, \pi_{2}-$ представления групп $G_{m}, G_{n-m}$, то через $\pi=\pi_{1} \circ \pi_{2}$ обозначается представление группы $G_{n}$, индуцированное тривиальным продолжением предста- 
вления $\pi_{1} \times \pi_{2}$ подгруппы $G_{m} \times G_{n-m} \subset P$ на параболическую подгруппу $P \subset G_{n}$. Здесь $P$ состоит из операторов в $V_{n}$, сохраняющих подпространство $V_{m}$. Аналогично определяется произведение нескольких представлений $\pi_{1} \circ \cdots \circ \pi_{s}$.

Неприводимое представление группы $G_{d}$, называется каспидальнылм, если оно не является подпредставлением никакого нетривиального параболического произведения представлений групп меньших степеней. Каспидальные представления группы $G L_{d}\left(\mathbb{F}_{q}\right)$ можно параметризовать точками множества $C_{d}(q)$. Заметим, что в число каспидальных представлений входят все характеры группы $G_{1} \cong \mathbb{F}_{q}^{*}$, в том числе единичный характер $e$, играющий в теории представлений группы $G L B$ особую роль.

Неприводимое представление группы $G_{n}$ называется $c$-npuмарныл, если оно является подпредставлением некоторой степени $c \circ \cdots \circ c$ каспидального представления $c$. Известно (см. $[5,6,10])$, что примарные представления параметризуются (при заданном $\left.c \in C_{d}(q)\right)$ множеством $\mathbb{Y}_{m}$ диаграмм Юнга c $m=n / d$ клетками. Обозначим через $\pi_{\lambda}^{c}$ неприводимое примарное представление группы $G_{m d}$, входящее в произведение $c \circ \cdots \circ c$ и отвечающее диаграмме Юнга $\lambda \in \mathbb{Y}_{m}$. Каждое неприводимое представление группы $G_{n}$ однозначно (с точностью до порядка сомножителей) представимо как параболическое произведение $\pi_{\varphi}=\prod_{c \in C} \pi_{\varphi(c)}^{c}$ дизъюнктных (т.е. входящих в степени различных каспидальных представлений) примарных представлений $\pi_{\varphi(c)}^{c}$, причем $n=\sum_{c}|\varphi(c)| d(c)$.

Таким образом, неприводимые представления $\pi_{\varphi}$ алгебры $A_{n}$ параметризуются семействами диаграмм Юнга $\varphi \in \Phi$ степени $n$, а вершины графа ветвления алгебр $A_{n}$ совпадают с вершинами графа $\Phi$. При этом каспидальные представления отвечают каспидальным семействам, примарные представления - семействам, в которых лишь одна из диаграмм непуста, а представления, дизъюнктные с единичным, соответствуют произвольным начальным семействам. Наша цель - проверить, что ветвление представлений алгебр $A_{n}$ совпадает с ветвлением в графе $\Phi$.

Пусть $N_{n} \subset G_{n+1}$ - группа операторов в $V_{n+1}$, сохраняющих $V_{n}$ и совпадающих на $V_{n}$ с тождественным оператором, а $P_{n}$ - группа операторов в пространстве $V_{n+1}$, сохраняющих $V_{n}$. Группа $P_{n}$ является полупрямым произведением подгруппы $G_{n} \subset G_{n+1}$ и нормального делителя $N_{n}$. Рассмотрим гомоморфизм $i: \mathbb{C}\left(G_{n}\right) \rightarrow \mathbb{C}\left(G_{n+1}\right)$, определяемый формулой $i(g)=\left|N_{n}\right|^{-1} \sum_{h \in N_{n}} g h$, как композицию вложения $i: \mathbb{C}\left(G_{n}\right) \rightarrow \mathbb{C}\left(P_{n}\right)$, заданного той же формулой, и гомоморфизма $\mathbb{C}\left(P_{n}\right) \rightarrow \mathbb{C}\left(G_{n+1}\right)$, задаваемого вложением групп $P_{n} \rightarrow G_{n+1}$.

Если $\pi$ - неприводимое представление группы $G_{n}$, то через $\widetilde{\pi}$ обозначим его продолжение на $P_{n}$, тривиальное на подгруппе $N_{n}$. Ясно, что $\tilde{\pi}(i(g))=$ $\pi(g)$ при любых $g \in G_{n}$. Если же неприводимое представление $\sigma$ группы $P_{n}$ нетривиально на $N_{n}$, то

$$
\sigma(i(g))=\frac{\sigma(g)}{\left|N_{n}\right|} \sum_{h \in N_{n}} \sigma(h)=0
$$

при всех $g \in G_{n}$. По теореме взаимности Фробениуса неприводимое представление $\tilde{\pi}$ группы $P_{n}$ содержится в ограничении на $P_{n}$ неприводимого 
представления $\pi_{\psi}$ группы $G_{n+1}$ в точности с той же кратностью, с какой $\pi_{\psi}$ входит в представление $\pi_{\varphi} \circ 1=\bigoplus_{\psi: \varphi \nearrow_{\psi}} \pi_{\psi}$, указанное разложение которого хорошо известно.

Обозначим через $\bar{A}$ алгебру $A$ с присоединенной единицей. Как и $A$, эта алгебра локально полупроста. Двусторонние идеалы алгебр $A$ и $\bar{A}$ совпадают. Напомним, что для алгебр без единицы диаграмма Браттели еще не определяет алгебру полностью - требуется указать размерности простых модулей. Диаграмма Браттели алгебры $\bar{A}$ с присоединенной единицей получается из графа $\Phi$ добавлением одной новой вершины $\delta_{n}$ на каждом этаже $\Phi_{n}, n=1,2, \ldots$. Новые вершины соседних этажей соединяются дугами без кратностей. Кроме того, из каждой вершины $\delta_{n}$ проводятся дуги подходящих кратностей во все прочие вершины следующего этажа. Заметим также, что дуги диаграммы алгебры $A$ не имеют кратностей, а диаграмма семейства алгебр $\left\{A_{n}\right\}$ получается из диаграммы Браттели семейства $\left\{G L_{n}(k)\right\}$ вычеркиванием некоторых дуг.

5. Структура алгебры $\boldsymbol{A}$. Имеется общая теория (см. [1,9]), которая описывает структуру локально полупростой алгебры, ее характеры, представления и группу Гротендика в терминах диаграммы Браттели этой алгебры. Опираясь на теорему 3, мы применяем эту теорию для получения основных фактов о группе $G L B$.

Если $\varphi \in \widetilde{\Phi}$ - начальное семейство и $\lambda$ - диаграмма Юнга, то через $\psi=\varphi \circ \lambda$ обозначим семейство диаграмм Юнга, для которого $\psi(e)=\lambda$ и $\psi(c)=\varphi(c)$ при $c \neq e$. По определению $\pi_{\varphi} \circ \lambda=\pi_{\varphi} \circ \pi_{\lambda}^{e}$, где $\pi_{\lambda}^{e}$ есть $e$-примарное представление, отвечающее диаграмме Юнга $\lambda$. Напомним, что через $e$ мы обозначаем выделенную точку множества $C_{1}$, параметризующую единичное представление группы $G L_{1}(k)$.

Обозначим через $A_{n}(\varphi)$ двусторонний идеал алгебры $A_{n} \cong \mathbb{C}\left(G L_{n}(k)\right)$, определенный как пересечение ядер всех неприводимых представлений $\pi_{\psi}$, $\psi \in \Phi_{n}$, отличных от $\pi_{\varphi}$. Для $\varphi \in \widetilde{\Phi}_{d}$ положим $I_{n}(\varphi)=\bigoplus_{\lambda} A_{n}(\varphi \circ \lambda)$ (сумма берется по всем $\left.\lambda \in \mathbb{Y}_{n-d}\right)$ и обозначим через $I(\varphi)=\bigcup_{n} I_{n}(\varphi)$ соответствующий двусторонний идеал в алгебре $A$.

Алгебра Гекке $H_{\infty}$ пары $(G L B, B)$ определяется как подалгебра тех функций $a \in A$, которые постоянны на двойных классах смежности по борелевской подгруппе $B$. Эта алгебра изоморфна групповой алгебре $\mathbb{C}\left(\mathfrak{S}_{\infty}\right)$ бесконечной симметрической группы. Подробности о характерах алгебры Гекке см. в [2].

Определение алгебры Гекке $H_{\infty}$, данное выше, параллельно классическому определению конечномерной алгебры Гекке $H_{n}$ как алгебры двойных классов по борелевской подгруппе в группе $G L_{n}(k)$ и эквивалентно современному определению с помощью бесконечного числа образующих и известных соотношений.

ТЕОРЕма 4. Алгебра $А$ есть сулмма счетного числа непересекаюшихся двусторонних идеалов $I(\varphi), \varphi \in \widetilde{\Phi}$. Каждый из этих идеалов стабильно изоморфен алгебре финитных функиий на бесконечной симметрической группе $\mathfrak{S}_{\infty}$. Идеал $I(\varnothing)$ содержит алгебру Гекке Н и стабильно изоморфен этой алаебре. 
6. Следы алгебры $\boldsymbol{A}$. Условимся обозначать через $\chi_{\varphi}$ след представления алгебры $A_{n} \cong \mathbb{C}\left(G L_{n}(k)\right)$, отвечающего семейству $\varphi \in \Phi_{n}$, нормированный условием $\chi_{\varphi}\left(a_{1}\right)=1$, где $a_{1}$ - единичный элемент алгебры $A_{1}$. Правило ветвления этих следов определяется формулой (1).

Определим симплекс Тома $\Delta$ как пространство пар вещественных неотрицательных невозрастающих последовательностей $\alpha=\left(\alpha_{1} \geqslant \alpha_{2} \geqslant \cdots \geqslant 0\right)$, $\beta=\left(\beta_{1} \geqslant \beta_{2} \geqslant \cdots \geqslant 0\right)$, для которых $\gamma \equiv 1-\sum \alpha_{i}-\sum \beta_{i} \geqslant 0$. Напомним [1], что точки симплекса Тома параметризуют неразложимые гармонические функции графа Юнга.

Для диаграммы Юнга $\lambda$ обозначим через $s_{\lambda}(\alpha ; \beta)$ функцию Шура, определенную на симплексе Тома $\Delta$, как указано в [1]. Если $g \in G_{n}$ и $\varphi \in \widetilde{\Phi}_{d}$ при $d \leqslant n$, то положим

$$
\chi_{\alpha, \beta, \varphi}\left(a_{g}\right)=\sum_{\lambda \vdash(n-d)} \chi_{\varphi \circ \lambda}(g) s_{\lambda}(\alpha ; \beta),
$$

где $\chi_{\varphi \circ \lambda}$ - характер неприводимого представления $\pi_{\varphi \circ \lambda}=\pi_{\varphi} \circ \pi_{\lambda}^{e}$ группы $G_{n}$. В силу правила ветвления и гармоничности функции $\lambda \mapsto s_{\lambda}(\alpha ; \beta)$ на графе Юнга функция $\chi_{\alpha, \beta, \varphi}$ корректно определена на алгебре $A$. Можно также записать

$$
\chi_{\alpha, \beta, \varphi}\left(a_{g}\right)=\sum_{\mu \vdash(n-d)}\left(\chi_{\varphi} \circ \chi_{\left(\mu_{1}\right)}^{e} \circ \cdots \circ \chi_{\left(\mu_{l}\right)}^{e}\right)(g) m_{\mu}(\alpha ; \beta),
$$

где $\chi_{\left(\mu_{1}\right)}^{e} \cdots \cdots \chi_{\left(\mu_{l}\right)}^{e}$ - произведение единичных характеров $\chi_{\left(\mu_{j}\right)}^{e}$ групп $G_{\mu_{j}}$, отвечающих отдельным строкам диаграммы Юнга $\mu=\left(\mu_{1}, \ldots, \mu_{l}\right)$, а $m_{\mu}(\alpha ; \beta)$ - мономиальные суперсимметрические функции от параметров Toma.

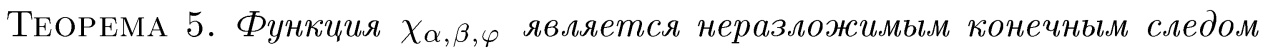
на алгебре $A$, нормированньм условием $\chi\left(a_{1}\right)=1$. Это общий вид всех таких следов.

Все следы алгебры $A$, кроме тривиального, неограниченны на аппроксимативной единице этой алгебры и поэтому определяют по известной конструкции ГНС представления алгебр $A$ и $\bar{A}$ бесконечного типа. Следы, отличающиеся от нуля только на идеале, содержащем алгебру Гекке, и соответствующие им представления мы называем унипотентными следами (соответственно факторпредставлениями). Последние можно описать как представления, в которых есть ненулевой вектор, инвариантный относительно борелевской подгруппы.

7. $\boldsymbol{K}_{0}$-функтор алгебры $\boldsymbol{A}$ и мультипликативность следов. В теории локально полупростых алгебр $K_{0}$-функтор (группа Гротендика) играет роль основного инварианта (см. [1]). Пусть $R_{n}=K_{0}\left(G_{n}\right)$ - группа Гротендика для $G_{n}=G L_{n}(k)$ и $R=\bigoplus_{n=0}^{\infty} R_{n}$. Параболическое индуцирование задает умножение, превращающее $R$ в ассоциативную алгебру. Кроме того, в $R$ имеется структура алгебры Хопфа, причем $R$ изоморфна как алгебра Хопфа тензорному произведению алгебр $\Lambda_{c}$ (см. 99 в [10]). Здесь $c \in C$ пробегает множество параметров каспидальных представлений $C$, а $\Lambda_{c}$ алгебра Хопфа симметрических полиномов от переменных $x_{1}^{c}, x_{2}^{c}, \ldots$ Всем 
этим переменным приписывается степень $d(c)$. Наша цель - связать $R$ с $K_{0}$-функтором алгебры $A$.

Обозначим через $\Lambda_{\varnothing}$ подалгебру, линейно порожденную представлениями

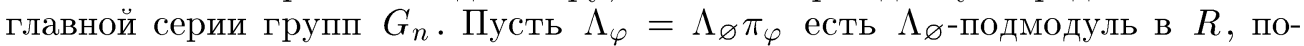
рожденный элементом $\pi_{\varphi} \in R$. Алгебра $R$ распадается в прямую сумму

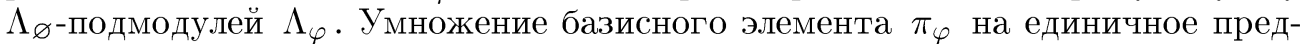
ставление $e \in R_{1}$ задает граф ветвления $\Phi$, который, таким образом, мультипликативен в смысле работы [1].

Следы алгебры $A$ естественно отождествляются с аддитивными функциями $f: R \rightarrow \mathbb{R}$, для которых $f\left(\pi_{\varphi}\right) \geqslant 0$ при всех $\varphi \in \Phi$ и $f(\pi \circ e)=f(\pi)$ при всех $\pi \in R$. Очевидно, что каждая такая функция есть сумма своих ограничений на подмодули $\Lambda_{\varphi}$. Поэтому каждая неразложимая функция отлична от нуля лишь на одном из элементов $\pi_{\varphi}, \varphi \in \bar{\Phi}_{d}$. С точностью до нормировки все функции $f_{\alpha, \beta, \varphi}$ с этим условием задаются формулой

$$
f_{\alpha, \beta, \varphi}\left(\pi_{\varphi \circ \lambda}\right)=s_{\lambda}(\alpha ; \beta),
$$

где $\pi_{\varphi} \in R_{d}$. Они отвечают следу $\chi_{\alpha, \beta, \varphi}$ алгебры $A$, заданному формулой (2).

ТЕОремА 6. Пусть ұ-характер главной серии факторпредставлений алгебры А. Пусть $g \in G_{m}, h \in G_{n-m}$ - два оператора, для которых все собственные числа (из алгебрачческого замыкания $\bar{k}$ поля $k=\mathbb{F}_{q}$ ) попарно различныл. Обозначим через $g \oplus h$ соответствуюший блочно-диагональньй оператор из $G_{n}$. Тогда справедлива формула $\chi\left(a_{g \oplus h}\right)=\chi\left(a_{g}\right) \chi\left(a_{h}\right)$.

\section{ЛИТЕРАТУРА}

1. Вершик A. M., Керов C. В. Локально полупростые алгебры. Комбинаторная теория и $K_{0}$-функтор. Итоги науки и техники. Совр. пробл. математики. Новейшие достижения. ВИНИТИ, т. 26, 1985, с. 3-56.

2. Вершик A. M., Керов C. В. Характеры и реализации представлений бесконечномерной алгебры Гекке и инварианты узлов. ДАН СССР, 301, № . 4, 777-780 (1988).

3. Джеймс Г. Теория представлений симметрических групп. Мир, М., 1982.

4. Макдональд И. Симметрические функции и полиномы Холла. Мир, М., 1985.

5. Фаддеев Д. K. Комплексные представления полной линейной группы над конечным полем. Записки научн. семинаров ЛОМИ, 46, 64-88 (1974).

6. Green J. A. The characters of the finite general linear groups. Trans. Amer. Math. Soc., 80, 402-447 (1955).

7. Skudlarek H. L. Die unzerlegbaren Charaktere einiger diskreter Gruppen. Math. Ann., 223, 213-231 (1976).

8. Thoma E. Die Einschränkung der Charactere von $G L(n, q)$ auf $G L(n-1, q)$. Math. Z., 119, 321-338 (1971).

9. Kerov S., Vershik A. The Grothendieck group of the infinite symmetric group and symmetric functions with the elements of the $K_{0}$-functor theory of $A F$-algebras. Adv. Stud. Contemp. Math., Gordon and Breach, Vol. 7, 1990, pp. 36-114.

10. Zelevinsky $A . V$. Representations of finite classical groups. Lecture Notes in Math., Vol. 869, 1981, pp. 1-184. 